\title{
Saúde sexual e infecções sexualmente transmissíveis na adolescência: Uma revisão integrativa
}

RESUMO | Objetivo: avaliar o conhecimento e o comportamento sexual dos adolescentes acerca das infecções sexualmente transmissíveis. Método: trata-se de uma revisão integrativa da literatura realizada nas bases de dados SCIELO, LILACS e BDENF por meio da estratégia PICO para definição da pergunta norteadora e do fluxograma PRISMA para seleção dos artigos. As buscas abrangeram o período de 2014 a 2019, sendo selecionados os artigos disponíveis em português, na íntegra e no formato original. Resultados: foram selecionados 10 artigos para a composição do estudo que demostraram déficit no conhecimento dos adolescentes acerca das infecções sexualmente transmissíveis, bem como não utilização do preservativo de modo rotineiro devido acreditarem que este inibe o prazer sexual. Conclusão: necessita-se orientar os adolescentes acerca das circunstâncias que acarretam às infecções sexualmente transmissíveis e sobre o uso correto do preservativo por meio de intervenções e educação em saúde nas escolas e nos serviços de saúde.

Palavras-chaves: Adolescente; Doenças sexualmente transmissíveis; Sexualidade.

ABSTRACT | TObjective: to evaluate the knowledge and sexual behavior of adolescents about sexually transmitted infections. Method: this is an integrative literature review performed in the databases SCIELO, LILACS and BDENF through the PICO strategy to define the guiding question and the PRISMA flowchart for article selection. The searches covered the period from 2014 to 2019, being selected the articles available in Portuguese, in full and in original format. Results: ten articles were selected for the composition of the study that showed a deficit in the adolescents' knowledge about sexually transmitted infections, as well as a nonuse of condoms on a routine basis because they believe that it inhibits sexual pleasure. Conclusion: adolescents need to be advised of the circumstances that lead to sexually transmitted infections and the correct use of condoms through health interventions and education in schools and health services.

Keywords: Adolescent; Sexually transmitted diseases; Sexuality.

RESUMEN | Objetivo: evaluar el conocimiento y el comportamiento sexual de los adolescentes sobre las infecciones de transmisión sexual. Método: esta es una revisión integradora de la literatura realizada en las bases de datos SCIELO, LILACS y BDENF a través de la estrategia PICO para definir la pregunta guía y el diagrama de flujo PRISMA para la selección de artículos. Las búsquedas abarcaron el período de 2014 a 2019, seleccionándose los artículos disponibles en portugués, en su totalidad y en formato original. Resultados: se seleccionaron 10 artículos para la composición del estudio que mostraron un déficit en el conocimiento de los adolescentes sobre las infecciones de transmisión sexual, así como la falta de uso de condones de forma rutinaria porque creen que inhibe el placer sexual. Conclusión: los adolescentes deben ser informados de las circunstancias que conducen a infecciones de transmisión sexual y el uso correcto de condones a través de intervenciones de salud y educación en escuelas y servicios de salud.

Descriptores: Adolescente; Enfermedades de transmisión sexual; Sexualidad.

\section{Letícia de Sousa Alves}

Enfermeira. Graduada pela Universidade Paulista (UNIP), Campus Brasília-DF, Brasil.

\section{Ricardo Saraiva Aguiar}

Enfermeiro. Professor Assistente. Curso de Graduação em Enfermagem, Universidade Paulista (UNIP), Campus Brasília-DF, Brasil.

Recebido em: 02/01/2020

Aprovado em: 30/01/2020

INTRODUÇÃO

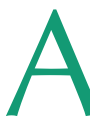
adolescência é uma etapa da vida onde ocorre a transição entre a infância e a idade adulta, sendo caracterizada por intenso crescimento e desenvolvimento. Além disso, apresenta uma das maiores incidências de infecções sexualmente transmissíveis (IST) ${ }^{1}$ devido, principalmente, aos comportamentos de risco ${ }^{2}$.

Para a Organização Mundial de Saúde (OMS), as IST estão entre as causas mais comuns de doenças no mundo e pode ser considerado um problema de saúde pública com várias consequências de natureza sanitária, social e econômi- ca devido a dificuldade de diagnóstico e tratamento precoce das mesmas, tendo como prognósticos graves sequelas como infertilidade, perda fetal, gravidez ectópica e morte prematura, bem como infecções em recém-nascidos e lactentes ${ }^{3}$.

Diante disso, os jovens necessitam de informações claras, apoio e compreensão acerca da temática. Nesse âmbito, a orientação sexual durante essa etapa de vida é imprescindível devido a necessidade de o adolescente adquirir a segurança necessária, perceber que sua vida sexual está se iniciando e que dispõe de amparo - seja da família, dos professores ou dos profissionais da saúde - para receberem informações corretas sobre o assunto ${ }^{4}$. 
Assim, as temáticas relacionadas à saúde sexual e reprodutiva devem ser abordadas de forma habitual por profissionais das áreas da saúde e educação junto aos adolescentes, por mais que o tema possa ser considerado cotidiano, o conhecimento de adolescentes acerca da temática ainda se apresenta como insuficiente ${ }^{5}$.

Portanto, o presente estudo tem o objetivo avaliar o conhecimento e o comportamento sexual dos adolescentes acerca das IST. Sob essa perspectiva, destaca-se a questão que norteou a presente proposta investigativa: qual a relação entre o conhecimento acerca das IST e o comportamento sexual dos adolescentes?

\section{MÉTODO}

Trata-se de uma revisão integrativa da literatura, uma vez que contribui no processamento sistemático e analítico dos resultados ${ }^{6}$.

Elaborou-se, na primeira fase, a pergunta norteadora de pesquisa utilizando a estratégia $\mathrm{PICO}^{7}$. Assim, considerou-se $P$ : pacientes adolescentes; I: saúde sexual; C: qualquer comparação entre saúde sexual; $\mathrm{O}$ : infecções sexualmente transmissíveis. Mediante a estratégia construída, elaborou-se a pergunta de pesquisa: qual a relação entre o conhecimento acerca das IST e o comportamento sexual dos adolescentes?

Organizou-se para a segunda fase, uma estratégia de busca por dois revisores independentes utilizando os descritores adolescente, doenças sexualmente transmissíveis e sexualidade nas bases de dados eletrônicas SCIELO, LILACS e BDENF.

Refinou-se, contemplando a terceira fase da pesquisa com a aplicação dos critérios de inclusão: artigos publicados de forma online no período de 2014 a 2019; disponíveis em língua portuguesa; na íntegra; e no formato original.

Na quarta fase foi lido criticamente os resumos dos estudos recuperados, excluindo os duplicados e aqueles cujo objetivo, resultados ou conclusão não mencionavam sobre a saúde sexual e o conhecimento dos adolescentes acerca das IST.

A extração dos dados foi conduzida por dois revisores independentes, sendo que os desacordos entre os revisores em relação aos dados extraídos foram discutidos, tendo como referência a publicação original.

\section{RESULTADOS}

Apresenta-se, na figura 1, o fluxograma descritor dos resultados obtidos a partir da estratégia de busca de acordo com o fluxograma PRISMA ${ }^{8}$.

Diante disso, no quadro 1 são apresentados os 10 artigos contidos nesta revisão integrativa, sendo interpretados e sintetizados todos os resultados, através de uma comparação dos dados evidenciados na análise dos artigos.
Figura 1. Fluxograma da seleção dos estudos. Brasillia, Distrito Federal, Brasil, 2019.

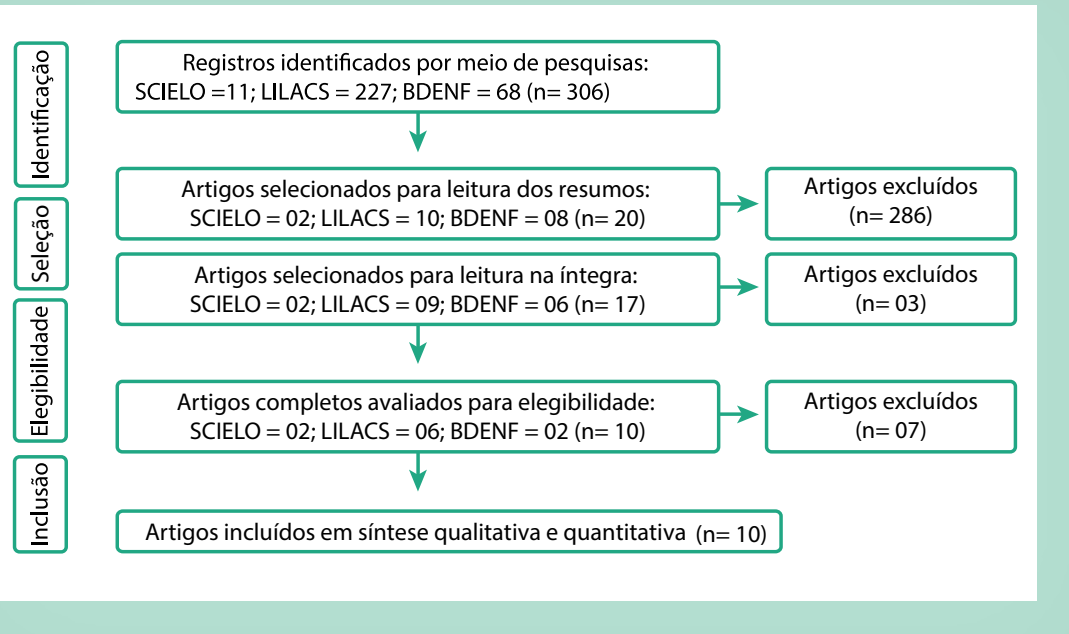

Quadro 1. Distribuição dos artigos. Brasilia, Distrito Federal, Brasil, 2019.

\begin{tabular}{|c|c|c|c|c|c|} 
& $\begin{array}{c}\text { Periódico e ano } \\
\text { de publicação }\end{array}$ & Autores & Título & Delineamento & $\begin{array}{c}\text { Nível de } \\
\text { Evidência9 }\end{array}$ \\
\hline Artigo 1 & $\begin{array}{c}\text { Revista de Enfer- } \\
\text { magem e Atenção } \\
\text { à Saúde, 2018 }\end{array}$ & $\begin{array}{c}\text { Silva SPC, Gui- } \\
\text { sande TCCA, } \\
\text { Cardoso AM }\end{array}$ & $\begin{array}{c}\text { Adolescentes em } \\
\text { conflito com a lei e a } \\
\text { vulnerabilidade para } \\
\text { IST/HIVIAIDS: conhe- } \\
\text { cimentos e vivências }\end{array}$ & $\begin{array}{c}\text { Estudo descritivo } \\
\text { com abordagem } \\
\text { qualitativa }\end{array}$ & $\begin{array}{c}\text { Observou-se a vulnerabilidade } \\
\text { como algo distante, embora } \\
\text { tivessem noção de que adotavam } \\
\text { comportamentos que os expu- } \\
\text { nham ao risco de contrair as IST. }\end{array}$ \\
\hline Artigo 2 & $\begin{array}{c}\text { Cogitare Enfer- } \\
\text { magem, 2018 }\end{array}$ & $\begin{array}{c}\text { Ferreira EA, Al- } \\
\text { ves VH, Pereira } \\
\text { AV et al }\end{array}$ & $\begin{array}{c}\text { Adolescentes no } \\
\text { espaço escolar e o } \\
\text { conhecimento a res- } \\
\text { peito da saúde sexual } \\
\text { e reprodutiva }\end{array}$ & $\begin{array}{c}\text { Estudo descritivo, } \\
\text { exploratório, de } \\
\text { natureza quali- } \\
\text { tativa }\end{array}$ & $\begin{array}{c}\text { Verificou-se que existe uma re- } \\
\text { lação direta entre o ato sexual } \\
\text { e o risco da gravidez indeseja- } \\
\text { da E do contágio por IST. }\end{array}$ \\
\hline
\end{tabular}




\begin{tabular}{|c|c|c|c|c|c|c|}
\hline Artigo 3 & $\begin{array}{l}\text { Semina: Ciências } \\
\text { Biológicas e da } \\
\text { Saúde, } 2017\end{array}$ & $\begin{array}{c}\text { Freire AKS, } \\
\text { Melo MCP, } \\
\text { Vieira MP et al }\end{array}$ & $\begin{array}{l}\text { Aspectos psicosso- } \\
\text { ciais da sexualidade } \\
\text { na adolescência: } \\
\text { diálogos e aprendiza- } \\
\text { gem na escola }\end{array}$ & $\begin{array}{l}\text { Estudo qualita- } \\
\text { tivo baseado na } \\
\text { pesquisa-ação e } \\
\text { na metodologia } \\
\text { da problemati- } \\
\text { zação }\end{array}$ & $\begin{array}{l}\text { Muitos adolescentes têm } \\
\text { receio de conversar com a } \\
\text { família sobre a sexualidade por } \\
\text { acreditarem que serão repre- } \\
\text { endidos, bem como têm pouco } \\
\text { conhecimento sobre IST e sobre } \\
\text { direitos sexuais e reprodutivos. }\end{array}$ & IV \\
\hline Artigo 5 & $\begin{array}{c}\text { Revista Brasileira } \\
\text { de Enfermagem, } \\
2017\end{array}$ & $\begin{array}{c}\text { Almeida RAAS, } \\
\text { Corrêa RGCF, } \\
\text { Rolim ILTP et al }\end{array}$ & $\begin{array}{l}\text { Conhecimento } \\
\text { de adolescentes } \\
\text { relacionados às } \\
\text { doenças sexualmente } \\
\text { transmissíveis e a } \\
\text { gravidez }\end{array}$ & $\begin{array}{l}\text { Estudo qualitativo } \\
\text { e descritivo }\end{array}$ & $\begin{array}{l}\text { O conhecimento sobre a } \\
\text { prevenção de IST e gravidez foi } \\
\text { principalmente relacionado ao } \\
\text { uso de preservativos, anticon- } \\
\text { cepcionais orais e adiamento do } \\
\text { início da atividade sexual. }\end{array}$ & IV \\
\hline Artigo 6 & $\begin{array}{c}\text { Texto \& Contexto } \\
\text { Enfermagem, } \\
2017\end{array}$ & $\begin{array}{c}\text { Genz N, Mein- } \\
\text { cke SMK, Carret } \\
\text { MLV et al }\end{array}$ & $\begin{array}{l}\text { Doenças sexualmen- } \\
\text { te transmissíveis: } \\
\text { conhecimento e } \\
\text { comportamento sexual } \\
\text { de adolescentes }\end{array}$ & $\begin{array}{l}\text { Estudo descritivo, } \\
\text { observacional de } \\
\text { caráter quanti- } \\
\text { tativo }\end{array}$ & $\begin{array}{l}\text { A maior parte dos adolescentes } \\
\text { souberam definir adequada- } \\
\text { mente o conceito de IST, sendo } \\
\text { apontado o uso de preser- } \\
\text { vativo como o método mais } \\
\text { eficaz para prevenção dessas } \\
\text { doenças. }\end{array}$ & IV \\
\hline Artigo 7 & $\begin{array}{c}\text { Revista Mineira } \\
\text { de Enfermagem, } \\
2017\end{array}$ & $\begin{array}{c}\text { Souza V, Pimen- } \\
\text { ta AM, Caetano } \\
\text { LC et al }\end{array}$ & $\begin{array}{l}\text { Conhecimentos, } \\
\text { vivências e crenças } \\
\text { no campo sexual: um } \\
\text { estudo com alunos } \\
\text { do ensino médio com } \\
\text { perfis socioeconômi- } \\
\text { cos diferenciados }\end{array}$ & $\begin{array}{l}\text { Estudo transver- } \\
\text { sal, descritivo e } \\
\text { analítico }\end{array}$ & $\begin{array}{c}\text { Os alunos da escola privada } \\
\text { tiveram maior domínio de co- } \\
\text { nhecimento no campo sexual, } \\
\text { reprodutivo e de prevenção } \\
\text { das IST do que os da escola } \\
\text { pública. }\end{array}$ & IV \\
\hline Artigo 9 & $\begin{array}{l}\text { Revista Brasileira } \\
\text { de Crescimento e } \\
\text { Desenvolvimento } \\
\text { Humano, } 2015\end{array}$ & $\begin{array}{l}\text { Gondim PS, } \\
\text { Souto FN, Mo- } \\
\text { reira CB et al }\end{array}$ & $\begin{array}{l}\text { Acessibilidade dos } \\
\text { adolescentes às fon- } \\
\text { tes de informações } \\
\text { sobre saúde sexual e } \\
\text { reprodutiva }\end{array}$ & $\begin{array}{l}\text { Estudo quanti- } \\
\text { tativo de caráter } \\
\text { descritivo }\end{array}$ & $\begin{array}{c}\text { Os adolescentes têm uma } \\
\text { acessibilidade relativamente } \\
\text { elevada a atividades educati- } \\
\text { vas na área de saúde sexual e } \\
\text { reprodutiva, sendo a escola o } \\
\text { principal espaço. }\end{array}$ & IV \\
\hline $\begin{array}{l}\text { Artigo } \\
10\end{array}$ & $\begin{array}{c}\text { Revista Enferma- } \\
\text { gem Uerj, } 2015\end{array}$ & $\begin{array}{c}\text { Penna LHG, } \\
\text { Rodrigues RF, } \\
\text { Ribeiro LV et al }\end{array}$ & $\begin{array}{l}\text { Sexualidade dos } \\
\text { adolescentes em situ- } \\
\text { ação de acolhimento: } \\
\text { contexto de vulnera- } \\
\text { bilidade para DST }\end{array}$ & $\begin{array}{l}\text { Estudo qualita- } \\
\text { tivo de caráter } \\
\text { descritivo-explo- } \\
\text { ratório }\end{array}$ & $\begin{array}{l}\text { Os cuidados de prevenção e } \\
\text { promoção da saúde sexual } \\
\text { tornam-se escassos e o diálogo } \\
\text { e a negociação pelo uso do } \\
\text { preservativo estão ausentes. }\end{array}$ & IV \\
\hline
\end{tabular}

Referente ao ano de publicação, foi constatado que a maior quantidade de ar- tigos publicados foi em 2017 com cinco (50\%), seguido de 2018 com dois (20\%),
2015 com dois (20\%) e 2016 com um artigo $(10 \%)$. O periódico de maior publi- 
cação foi a Revista de Enfermagem Uerj com três artigos (30\%). Quanto à profissão do primeiro autor, em nove (90\%) a primeira autoria era de enfermeiro e em um $(10 \%)$ não foi encontrado informações específicas relacionadas à sua formação, pois se apresentava somente a vinculação acadêmica.

Quanto à região do país em que as pesquisas foram realizadas, cinco $(50 \%)$ foram desenvolvidas na região Nordeste, três $(30 \%)$ na região Sudeste, uma $(10 \%)$ na região Norte e uma (10\%) na região Sul.

Sobre o local de realização do estudo, foi identificado que em um foi realizado no âmbito da atenção terciária à saúde (10\%) e em nove fora dos níveis de atenção, sendo sete (70\%) em escolas, um $(10 \%)$ em Centro de Atendimento Socioeducativo e um (10\%) em Unidade de Acolhimento aos Adolescentes em Conflito com a Lei.

\section{DISCUSSÃO}

A sexualidade, especialmente na adolescência, é um componente intrínseco e fundamental na saúde dos jovens porque transcende o aspecto meramente biológico, manifestando-se também como um fenômeno psicológico e social, sendo influenciados por crenças e valores pessoais, familiares e normas morais ${ }^{10}$.

É nesta fase que a sexualidade assume papel de importância na identidade dos adolescentes através da manifestação dos desejos e da atração sexual. ${ }^{11}$ Assim, a preocupação com a saúde sexual e reprodutiva desse público é devido a precocidade do início da atividade sexual, a qual coopera para expô-los aos riscos de gravidez não planejada e IST ${ }^{12-14}$.

Nesse contexto, a escola é um local estratégico de cuidado aos adolescentes, os quais devem ser estimulados a participarem de projetos que informem a respeito da saúde sexual e reprodutiva. A escola é um local privilegiado para socializar, além de ser um espaço em que muitos adolescentes desejam que aconteça a educação em saúde a respeito das
IST e da gravidez. Dessa forma, observa-se, atualmente, que programas e projetos sexuais são fundamentais para atender o adolescente quanto a sua sexualidade ${ }^{12}$.

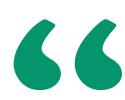

Nesse contexto, a escola é um local estratégico de cuidado aos adolescentes, os quais devem ser estimulados a participarem de projetos que informem a respeito da saúde sexual e reprodutiva.

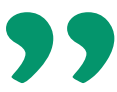

Contudo, a família é a uma das principais reguladoras da sexualidade e das informações, pois geralmente se limitam a ditar regras de comportamento que estão relacionadas a valores para manutenção do sistema familiar. Assim, identifica-se que existe a necessidade da formação de uma rede social de apoio aos adolescentes, às famílias e à escola a fim de que eles recebam informações corretas ${ }^{12}$.
Diante disso, um dos assuntos mais explorados durante uma dinâmica com adolescentes foi à virgindade, considerada um tema de difícil abordagem e que requer confiança para ser compartilhado, principalmente entre amigos. Ademais, foi identificado déficit no conhecimento a respeito do uso do preservativo feminino e maior conhecimento sobre o masculino, porém foi notável o despreparo entre ambos os sexos no momento de colocar os preservativos em próteses evidenciando a necessidade de mais orientações de caráter preventivo e educacional, principalmente no âmbito das escolas ${ }^{15}$.

No que se refere à frequência atual do uso de métodos para evitar a gravidez, pouco mais da metade dos jovens referiram sempre utilizar método contraceptivo. Entre os que faziam uso da contracepção, 46\% usavam apenas preservativo e $29 \%$ usavam-no associado a outro método (dupla proteção) ${ }^{16}$.

Sobre as IST, identificou-se que os adolescentes citaram mais o HIV (>90\%), herpes genital ( $>60 \%$ ), sífilis $(>60 \%$ ) e a gonorreia $(>55 \%)$ como as mais comuns de transmissão via sexual. Entretanto, chamou atenção o desconhecimento de outras IST prevalentes, como a tricomoníase, hepatite $\mathrm{B}$, candidíase e HPV ${ }^{17}$.

Quanto a responsabilidade pela prevenção das IST, verificou-se que 96,6\% dos adolescentes achavam que competia tanto ao homem quanto à mulher; $2 \%$ referiram ser responsabilidade do homem; $0,8 \%$ achavam que a responsabilidade era da mulher; e $0,6 \%$ não soube informar ${ }^{18}$.

Na perspectiva da educação em saúde, sentir-se à vontade para conversar sobre a vida sexual com a mãe apresenta associação positiva com o uso consistente de contraceptivos, mas o professor constitui a primeira opção entre os adolescentes sobre as fontes de informação acerca das IST. Nesse contexto, evidencia-se a importância por parte do Estado e da comunidade escolar na busca de uma educação que contemple essa temática na sua transversalidade, sendo importante que os pais não deleguem a outros a 
tarefa de falar com os filhos sobre sexo ${ }^{13}$.

Contudo, os serviços de saúde também devem garantir acesso à informação e educação em saúde sexual e reprodutiva por meio de métodos que auxiliem os adolescentes a evitarem gravidez não planejada e a prevenirem-se contra IST. Assim, é imprescindível as orientações antes mesmo do início das práticas sexuais para ajudá-los a lidarem com a sua sexualidade de forma positiva e responsável, incentivando comportamentos de prevenção e de autocuidado. ${ }^{16}$ Desse modo, a enfermagem no papel de educador é de fundamental importância para a promoção da saúde sexual e reprodutiva de modo saudável ${ }^{13}$.
Portanto, trabalhar a educação sexual e reprodutiva com adolescentes significa ampliar e aprofundar a visão sobre a sexualidade, ressaltando os diferentes pontos de vista existentes na sociedade, incluindo as práticas sexuais ligadas ao afeto, prazer, ao respeito e à própria sexualidade. Por conseguinte, a orientação sexual deve compreender o desenvolvimento sexual como saúde reprodutiva, relações de gênero, relações interpessoais, afetivas, imagem corporal e autoestima ${ }^{10}$.

\section{CONCLUSÃO}

Observa-se que há um déficit no conhecimento dos adolescentes sobre IST e gravidez precoce, necessitando de intervenções que proporcionem a prevenção das IST e da gravidez não planejada. Os adolescentes têm conhecimento que o preservativo é o método principal para evitar a disseminação das IST, porém não utilizam por acreditarem que inibe o prazer sexual. Além disso, identificou-se que há o reconhecimento de algumas IST por parte dos adolescentes, como o HIV, herpes, sífilis e gonorreia.

Nessa perspectiva, a educação em saúde é primordial, começando pela família e, principalmente, no âmbito escolar e nos serviços de saúde para a garantia de autonomia e consequentemente uma maior prática de autocuidado aos adolescentes.

\section{Referências}

1. Costa ID, Nunes NNS. Compreensão dos adolescentes sobre a prevenção e transmissão das infecções sexualmente transmissiveis em escolas do município de presidente Médici. Acta Biomed Bras. 2017;8(1):12-23. Disponível em: https://www.actabiomedica.com.br/index.php/acta/article/view/161 2. Oliveira FA, Queiroz AM, Chaves MAS, Branco MFCC, Mendes IC. Atividades lúdicas desenvolvidas com adolescentes escolares sobre prevenção de infecções sexualmente transmissiveis. Rev Interd. 2017;1(3):53-63. Disponível em: https:// revistainterdisciplinar.uninovafapi.edu.br/index.php/revinter/article/view/1124 3. Cortez EA, Silva LM. Pesquisa-ação: promovendo educação em saúde com adolescentes sobre infecção sexualmente transmissivel. Rev enferm UFPE online. 2017;11(9):3642-9. Disponivel em: http://bases.bireme.br/cgi-bin/ wxislind.exe/iah/online/?IsisScript=iah/iah. $x i s \& s r c=$ google\&base=BDENF\&lang=p\&nextAction=Ink\&exprSearch $=33164$ \&indexSearch=ID 4. Queiroz VR, Almeida JM. Sexualidade na adolescência: potencialidades e dificuldades dos professores de ensino médio de uma escola estadual de Sorocaba. Rev Fac Cienc Med Sorocaba. 2017;19(4):209-14. Disponível em: http://ken.pucsp.br/RFCMS/article/view/31788/pdf

5. Oliveira PC, Pires LM, Junqueira ALN, Vieira MAS, Matos MA, Caetano KAA et al. Conhecimento em saúde sexual e reprodutiva: estudo transversal com adolescentes. Rev eletrônica enferm. 2017;19:a17. Disponível em: https://revistas.ufg.br/fen/article/view/39926

6. Aguiar RS, Santana DC, Santana PC. A percepção do enfermeiro da estratégia saúde da família sobre a saúde do homem. Rev enferm Cent-Oeste Min. 2015;5(3):1844-54. Disponível em: http://www.seer.ufsj.edu.br/index. $\mathrm{php} / \mathrm{recom} /$ article/view/872

7. Donato $H$, Donato $M$. Etapas na condução de uma revisão sistemática. Acta Med Port. 2019;32(3):227-235. Disponivel em: https://www.actamedicaportuguesa.com/revista/index.php/amp/article/view/11923/5635 8. Moher D, Liberati A, Tetzlaff J, Altman DG, The PRISMA Group. Preferred reporting items for systematic reviews and meta-analyses: the PRISMA statement PLoS Med. 2009;6(7):e1000097. Disponível em: www.prisma-statement.org 9. OCEBM Levels of Evidence Working Group. The Oxford 2011 Levels of Evidence. Oxford: Oxford Centre for Evidence-Based Medicine; 2011. Disponível em: https://www.cebm.net/2016/05/ocebm-levels-of- evidence/ 10. Ferreira EA, Alves VH, Pereira AV, Rodrigues DP, Paiva ED, Santos IMM. Adolescentes no espaço escolar e o conhecimento a respeito da saúde sexual e reprodutiva. Cogitare enferm. 2018;23(2):e55851. Disponível em: https://revistas.ufpr.br/cogitare/article/view/55851

11. Penna LHG, Rodrigues RF, Ribeiro LV, Paes MV, Guedes CR. Sexualidade dos adolescentes em situação de acolhimento: contexto de vulnerabilidade para DST. Rev enferm UERJ. 2015;23(4):507-12. Disponivel em: https:// www.e-publicacoes.uerj.br/index.php/enfermagemuerj/article/view/18402 12. Gondim PS, Souto FN, Moreira CB, Cruz MEC, Caetano FHP, Montesuma FG. Acessibilidade dos adolescentes às fontes de informações sobre saúde sexual e reprodutiva. Rev bras crescimento desenvolv hum. 2015;25(1):50 53. Disponível em: http://pepsic.bvsalud.org/scielo.php?script=sci_arttext\&pid=S010412822015000100006\&lng =pt\&nrm=iso\&tlng=pt

13. Almeida RAAS, Corrêa RGCF, Rolim ILTP, Hora JM, Linard AG, Coutinho NPS et al. Conhecimento de adolescentes relacionados às doenças sexualmente transmissíveis e a gravidez. Rev bras enferm. 2017;70(5):1087-94. Disponível em: http://www.scielo.br/scielo.php?pi$\mathrm{d}=$ S0034-71672017000501033\&script=sci_arttext\&tlng=pt

14. Silva SPC, Guisande TCCA, Cardoso AM. Adolescentes em conflito com a lei e a vulnerabilidade para IST/HIV/AIDS: conhecimentos e vivências. Rev enferm atenção saúde. 2018;7(2):95-108. Disponível em: http://seer.uftm. edu.br/revistaeletronica/index.php/enfer/article/view/2384

15. Freire AKS, Melo MCP, Vieira MP, Gomes IM, Gomes JL, Ribamar DS et al. Aspectos psicossociais da sexualidade na adolescência: diálogos e aprendizagem na escola. Semina cienc biol saude. 2017;38(1):3-14. Disponivel em: http://www.uel.br/revistas/uel/index.php/seminabio/article/ view/26736/22629

16. Maciel KMN, Andrade MS, Cruz LZ, Fraga CDS, Paixão GPN, Souza RS Caracterização do comportamento sexual entre adolescentes. Rev enferm UERJ. 2017;25:e23496. Disponível em: https://www.e-publicacoes.uerj.br/ index.php/enfermagemuerj/article/view/23496

17. Souza V, Pimenta AM, Caetano LC, Cardoso JSR, Beinner MA, Villela LCM. Conhecimentos, vivências e crenças no campo sexual: um estudo com alunos do ensino médio com perfis socioeconômicos diferenciados. REME rev min enferm. 2017;21:e991. Disponivel em: http://www.reme.org.br/artigo/detalhes/1127 18. Genz N, Meincke SMK, Carret MLV, Corrêa ACL, Alves CN. Doenças sexualmente transmissíveis: conhecimento e comportamento sexual de adolescentes. Texto \& contexto enferm. 2017;26(2):e5100015. Disponível em: http://www.scielo.br/scielo.php?pid=S0104=07072017000200311-\&scriptsci_abstract\&tlng=pt 